\title{
Dinámica de sistemas en la gestión de inventarios
}

\section{Dynamics of systems in the management of inventories}

\author{
Daniel Alejandro Agudelo Serna \\ Universidad de San Buenaventura \\ alejo.1296@hotmail.com
}

\author{
Yohana Marcela López Rivera \\ Universidad de San Buenaventura \\ Yohana.lopez@usbmed.edu.co
}

(Tipo de Artículo: Revisión. Recibido: 09/11/2018. Aprobado: 06/12/2018)

\begin{abstract}
Resumen. En el presente artículo se estudia el proceso de gestión de inventarios por medio de la dinámica de sistemas, como herramienta metodológica que permite conocer el comportamiento de las variables de acuerdo a sus relaciones y a las decisiones que se tomen teniendo en cuenta su causalidad y realimentación. El desarrollo del documento está determinado por una revisión de conceptos teóricos relacionados con la gestión de inventarios y dinámica de sistemas, seguido por una revisión de literatura, en donde se analizan modelos dinámicos que relacionan las variables que afectan directa o indirectamente al inventario, resaltando la importancia de modelos robustos que permitan obtener mejores resultados y así poder establecer políticas de gestión y control del inventario. El objetivo fundamental es presentar una revisión de los modelos dinámicos relacionados con el inventario, en donde es posible observar los diagramas causales y de Forrester, variables, criterios y parámetros que influyen en cada uno de los sistemas presentados. Se supone una metodología basada en la búsqueda de información, para posteriormente desarrollar un análisis detallado de cada modelo encontrado.
\end{abstract}

Abstract. In the present article the process of inventory management is studied through the dynamics of systems, as a methodological tool that allows to know the behavior of the variables according to their relationships and to the decisions that are taken taking into account their causality and feedback. The development of the document is determined by a review of theoretical concepts related to the management of inventories and systems dynamics, followed by a review of the literature, where dynamic models are analyzed that relate the variables that directly or indirectly affect the inventory, highlighting the importance of robust models that allow obtaining better results and thus be able to establish inventory management and control policies. The main objective is to present a review of the dynamic models related to the inventory, where it is possible to observe the causal and Forrester diagrams, variables, criteria and parameters that influence each of the systems presented. It is assumed a methodology based on the search of information, to later develop a detailed analysis of each model found.

Palabras clave. Dinámica de sistemas, gestión de inventarios, simulación.

Keywords. System dynamics, inventory management, simulation.

DOI: $10.21500 / 20275846.3305$

\section{Introducción}

La cadena de suministro implica un proceso logístico que tiene en cuenta actividades como el control de inventarios, transporte y la transformación de materias primas [1]. Permitiendo a las organizaciones ofrecer productos y servicios bajo los requerimientos $y$ necesidades del cliente, teniendo en cuenta la calidad de los productos y la generación de un valor agregado, lo que permite adquirir a la organización una ventaja competitiva.

El funcionamiento de la cadena de suministro se establece en la secuencialidad de los eslabones, siempre en miras de satisfacer las necesidades del cliente final. Pero se olvida lo que hay más allá de este proceso y es justamente donde la mala planeación y la toma de decisiones afectan los procesos y actividades trayendo consecuencias en los costos [2].
En la cadena de suministro va a estar presente la administración de los inventarios [3] ya que permite tener un control sobre los procesos que se desarrollan en la empresa, desde que se adquiere la materia prima hasta que el producto terminado llega al cliente final [4]. Pero administrar el inventario es una tarea difícil que requiere de una correcta gestión para mitigar el impacto de la demanda y los riesgos que se puedan presentar en la relación empresa-cliente. En México, por ejemplo, setenta de cada cien Pymes no sobreviven por un tiempo mayor a 5 años [5] [6], debido a que las empresas no tienen control en el manejo del inventario, ni cuentan con herramientas de apoyo. Unas situaciones similares enfrentan las empresas cubanas, quienes tampoco tienen herramientas para predecir la demanda $u$ otros comportamientos. En Estados Unidos, en el año 2017 según [7] la representación del costo del inventario se encuentra entre el $30 \%$ y el $35 \%$ del valor de la empresa. Adicionalmente, la acumulación de inventario de los 
minoristas ha generado que las empresas norteamericanas hayan aumentado el tiempo para liquidar sus existencias a 1,38 meses [8].

En Colombia se presentan casos similares en las grandes, medianas y pequeñas empresas con relación al manejo del inventario bien sea de materias primas, producto en proceso o producto terminado [9]. Como se afirma en [10], el 78,3\% de los negocios que se abren el Colombia sobreviven un año, lo que evidencia que las empresas necesitan mejorar estratégicamente en la construcción de políticas de inventario para sobrevivir y mantenerse en el mercado. Como se expresa en [11], la empresa Fabricato detuvo su actividad productiva durante 15 días, dado a que las unidades almacenadas en inventario de producto terminado excedieron la capacidad de almacenamiento y de operación de la empresa, impactando negativamente en el ebitda.

Es evidente que el manejo ineficiente de los costos en el inventario trae consigo un decaimiento en la productividad de la empresa, afecta su ventaja competitiva, e incluso se asocian incrementos en los costos de mantener un inventario.

La modelación de los sistemas dinámicos ha sido de gran utilidad hoy en día, ya que es posible realizar un análisis según la complejidad del sistema en cuanto al comportamiento de las variables que influyen en un determinado proceso o actividad [12]. Entendiendo entonces la dinámica de sistemas como una herramienta para controlar y manejar de forma eficiente todos los sistemas y operaciones por medio de simulaciones que permitan ver el comportamiento y los cambios del proceso en el tiempo [13]. Esto lleva a que, por medio de la dinámica de sistemas, sea posible conocer el comportamiento de todo lo que influye en la administración del inventario para mejorar la productividad, disminuir costos, y aumentar la ventaja competitiva empresarial.

Este artículo presenta una revisión de literatura en relación al uso de la dinámica de sistemas como herramienta de control y administración del inventario, por medio de modelos sistemáticos en donde se ven influenciadas variables, criterios y parámetros con relación al inventario. Permitiendo realizar un análisis con relación a la toma de decisiones y los impactos generados con la modelación sistémica de los inventarios.

\section{Gestión de Inventarios}

En el entorno empresarial el control de los inventarios juega un papel muy importante, puesto que es una de las actividades logísticas en donde es posible encontrar una alta probabilidad de reducir los costos de las empresas [14]. Para el manejo y control de los inventarios existen múltiples modelos y herramientas para llevar un seguimiento y permitir la reducción de los costos.

Inventario: hace referencia a la verificación y control de los materiales o bienes de la empresa de modo que se pueda regular las existencias con que se cuentan[15] . También, de acuerdo con Ballou [4], "Los inventarios son acumulaciones de materias primas, provisiones, componentes, trabajo en proceso y productos terminados que aparecen en numerosos puntos a lo largo del canal de producción y logística de una empresa". El inventario se ve afectado directamente por la demanda ya que es posible que algunos productos tengan una alta variación en de la demanda con respecto a otros.

El objetivo principal del inventario es confirmar o verificar algún tipo de existencia en la empresa[15]. Según Wild, como se menciona en [16], el propósito del control del inventario es asegurar el funcionamiento de las actividades de la empresa, mediante la optimización basada en tres objetivos principales: Servicio al cliente, costo de inventario y costos operativos. Con estos tres, es posible tener un control sobre el inventario llevando a que la empresa conozca la disponibilidad de materiales en diferentes locaciones o actividades que desarrolle la empresa en relación a la cadena de abastecimiento.

A partir del objetivo principal del inventario, es posible identificar las funciones que este tiene, que según Donald J. Bowersox [1] y Heizer [17] es posible mencionarlas de la siguiente manera:

- Especialización geográfica: Este permite una distribución en la empresa de acuerdo a la fabricación. Conservando los lugares, etapas del proceso y creación, permitiendo la creación de valor en la especialización

- Desacoplamiento: Permite separar las diferentes partes del proceso productivo. De modo que cada proceso opere de manera eficiente.

- Equilibrio suministro/demanda: Atiende el tiempo transcurrido entre la disponibilidad del inventario (fabricación, abasto, desabasto) y el consumo.

- Disminución de la incertidumbre: Se relaciona con la incertidumbre de la demanda en exceso y los retrasos inesperados en el proceso de recepción.

Las funciones del inventario deben ser aplicadas a cualquier tipo o clasificación de inventario, ya que esto permite el mejoramiento de las condiciones de control del inventario, de modo que se pueda tomar acciones y llevar un seguimiento de acuerdo al tipo de inventario que se esté manejando.

La determinación del tipo de inventario es importante, ya que es posible asegurar el funcionamiento de la empresa de acuerdo a las actividades que en esta se desempeñen. Según [15][16] [17] el inventario se podría clasificar en: Materias primas, trabajo (producto) en proceso, suministro de mantenimiento, reparación y operación y finalmente el inventario en producto terminado.

El manejo de estos tipos de inventarios tiene un costo asociado de mantenimiento, en los cuales se tiene en cuenta aquellos que resultan de guardar, mantener, almacenar artículos durante un periodo de tiempo y son proporcionales a la cantidad promedio de artículos 
disponibles [4]. Estos costos pueden ser aquellos relacionados con: el capital, impuestos, seguro, obsolescencia, almacenamiento, entre otros. También se conoce el costo de ordenar y finalmente con estos es posible determinar el costo total del inventario.

La gestión de inventario es un proceso que involucra variables con las cuales es posible determinar el comportamiento a partir de la toma de decisiones y así evidenciar el comportamiento de los costos. Esto es posible entenderlo gracias a la dinámica de sistemas.

\subsection{Dinámica de sistemas}

La dinámica de sistemas es una metodología ideada para analizar y simular problemas en tiempo real de modo que se pueda comprender el comportamiento del sistema por medio de interacciones que serán las que determinen cambios en el sistema [12]. La aplicación de la dinámica de sistemas busca relacionar variables entorno a una decisión tomada, y se ha extendido a muchos campos tales como el político, medioambiental, medicina, ingeniería, así como en otros campos ya que el estudio de esta metodología permite mostrar el cambio de las cosas en el tiempo [18].

El pensamiento sistémico busca comprender esas relaciones que conforman un sistema para orientar a resultados de modo que los patrones y variables resulten más claros y puedan ayudar a estudiar los sistemas [18][19]

Por medio de la dinámica de sistemas, desarrollada por J. Forrester, es posible estudiar el comportamiento de los sistemas por medio de unas características de realimentación de modo que se logre una integración en los flujos de información del sistema [20].

Se conoce como sistema dinámico aquel en donde su salida en el presente depende de una entrada en el pasado; si su salida en curso depende solamente de la entrada en curso, el sistema se conoce como estático. La salida de un sistema estático permanece constante si la entrada no cambia y cambia solo cuando la entrada cambia. Por otro lado, en el sistema dinámico la salida va a cambiar con el tiempo cuando el sistema no está en equilibrio [21].

Para construir un modelo dinámico, es necesario analizar el sistema que se va a modelar y definir el problema. Sin embargo, en caso de tener un sistema más complejo es posible recurrir a una metodología más detallada para representar un sistema dinámico. Según [13] y [22] es posible construir un modelo dinámico como se muestra en la figura 1:

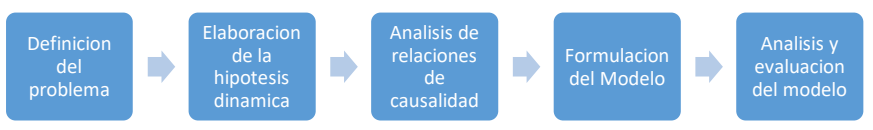

Figura 1. Pasos para la construcción de un modelo dinámico
El planteamiento del problema es la primera fase del proceso de construcción del modelo dinámico, en donde una de sus principales actividades es explorar el área de estudio y definir las preguntas que van a orientar el estudio [23]. La hipótesis dinámica busca relacionar lo teórico con una situación ideal. Esta normalmente se presenta por medio de un diagrama causal, en donde es posible relacionar todas las variables. Este diagrama presenta el conjunto de relaciones entre los elementos, presentando información concreta de los flujos y niveles, siendo así, una herramienta útil que permite conocer la estructura de realimentación del sistema por medio de bucles de realimentación en donde se causa un efecto directo o indirecto sobre las variables de estudio del modelo [24][25] [26].

\subsection{Revisión de literatura}

Los costos relacionados con el manejo y control del inventario han tomado gran importancia en las organizaciones, debido a que estos debilitan los eslabones de la cadena de suministro impactando negativamente el flujo normal de producción. De este modo, se hace necesario equilibrar los sistemas de gestión de la demanda, de modo que sea posible tener un control sobre los inventarios y así reducir los niveles de incertidumbre [27].

Cuando se analiza un inventario, es posible determinar su comportamiento a partir de factores y variables con las cuales sea posible controlar los costos, logrando equilibrar los datos en existencia [28]. Por tal motivo, para realizar este proceso es necesario proponer métodos en los cuales se pueda garantizar un equilibrio en las unidades de existencia y de este modo los costos también se disminuyan llevando a que la ventaja competitiva de la empresa mejore. De este modo es posible que se desarrollen otras metodologías con las cuales se mejore el proceso de gestión de inventarios, ya que estos juegan un papel muy importante en cualquier entidad económica, logrando una mejor satisfacción en los clientes [29], ya que normalmente se utilizan técnicas como el EOQ, con la cual se obtiene la cantidad optima de pedido, tiempos entre pedido, costos, entre otros; además de técnicas y metodologías de la investigación de operaciones y economía.

La dinámica de sistemas, juega un papel muy importante en la gestión de inventarios, ya que es una de las metodologías con las cuales es posible observar el comportamiento del inventario, ayudando en la toma de decisiones a partir de los cambios en la demanda [30]. Con esta herramienta es posible determinar, por medio de una simulación, cual es el comportamiento que están enfrentando ciertas variables frente a ciertos estímulos.

La construcción de modelos que permitan evaluar el comportamiento del inventario, a partir de la dinámica de sistemas, confluyen en sus principios fundamentales, los cuales pretenden mostrar cómo cambian las variables con el paso del tiempo y llevar a un acercamiento matemático, de modo que el sistema brinde soluciones 
analíticas a situaciones de alta complejidad [31]. A continuación, se presentarán modelos que han sido desarrollados para analizar y estudiar el comportamiento de los inventarios a partir de la dinámica de sistemas como herramienta metodológica de análisis.

Cuando se estudia un sistema de inventarios, es importante reconocer que existen excesos, retrasos y dificultades en la toma de decisiones. A partir de esto, es necesario establecer políticas a partir de representaciones en el espacio (modelos), que logren estabilizar el sistema de gestión de inventarios. Los modelos que se presentan en [32], inicialmente comprende de un modelo simple de realimentación del inventario por un flujo de entrada proveniente de una línea de suministro. Este modelo tiene únicamente una variable de nivel, que es el inventario, y dos variables de flujo. El inventario es alimentado por una tasa de adquisición TA, la cual para este caso no presenta ningún retraso, y es evidente que al no tener retraso el modelo se hace muy poco realista. El flujo de salida o tasa de pérdida (TP), depende del tiempo de vida promedio que tenga el producto. En la figura 2, se muestra la estructura inicial del modelo de inventario:

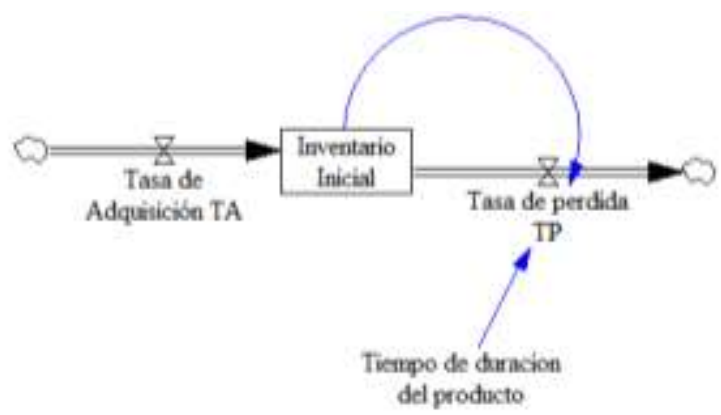

Figura 2. Estructura del inventario inicial. Fuente: Adaptado de [32]

Para llevar el modelo a un entorno más realista, es necesario tener en cuenta los retrasos en la tasa de adquisición. Como se muestra en la figura 3, el modelo poco a poco va adquiriendo nuevas políticas y lo hace mucho más cercano a la realidad.

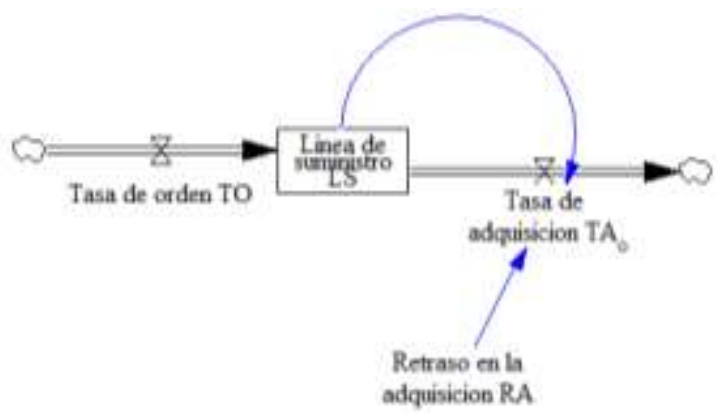

Figura 3. Estructura de la línea de suministro. Fuente: Adaptado de [32]

Las ecuaciones del modelo de inventario y línea de suministro, presentados anteriormente, están regidas por:

$$
\begin{gathered}
\frac{d I}{d t}=T A-T P \\
\frac{d L S}{d t}=T O-T A
\end{gathered}
$$

Con estos dos modelos simples, se buscó establecer políticas de gestión para cada uno de ellos de manera independiente, logrando determinar que la tasa de adquisición es no negativa, es decir, que no hay devoluciones, ni descartes. Además de esto, como se muestra en la figura 4 , se hacen modificaciones a la línea de suministro y al inventario, debido a que cuando se presentan diferencias en el inventario y el inventario deseado, la estabilidad del modelo cambia. Por dicha razón es necesario implementar una tasa esperada de pérdida, lo que lleva a que la tasa esperada de pérdida (TEP) sea igual a la tasa de pérdida (TP).

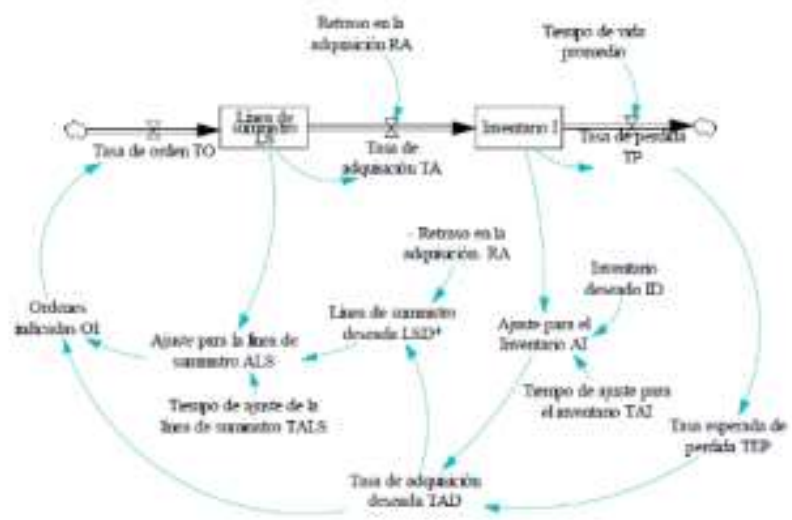

Figura 4. Modelo de Sterman. Fuente: [32]

A partir de los modelos presentados anteriormente, se hacen modificaciones a las políticas de gestión del inventario, construyendo independientemente políticas para el inventario y para la línea de suministro, donde posteriormente se procede a la construcción de un único modelo, como el que se observa a continuación:

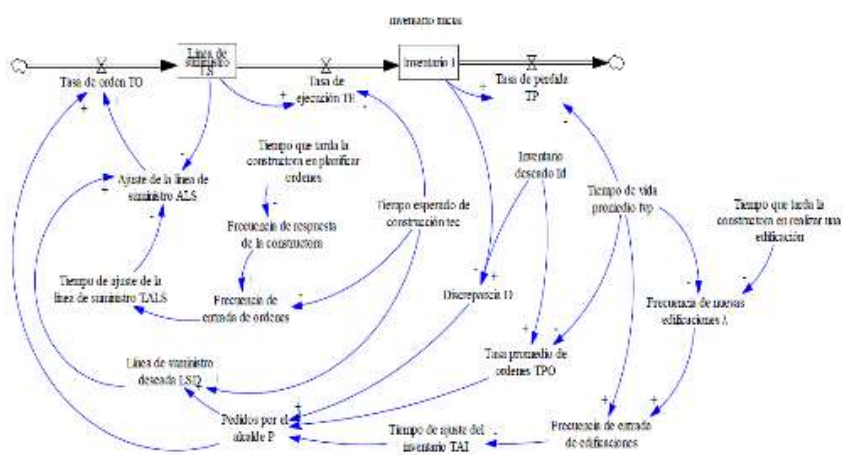

Figura 5. Modelo Inventario- línea de suministro (I-LS). Fuente: [32]

Ahora bien, es posible tener los dos modelos, es decir, las dos variables de nivel, y a partir de ello, hacer un modelo conjunto en donde se reúnan las variables de ambos modelos y se logren determinar políticas. En este caso la línea de suministro e inventario, son gestionados 
como un mismo sistema. Lo que indica que tiene dos retrasos y serán los reguladores de las órdenes, es decir, cuándo se debe ordenar nuevamente. Como se observa en la figura 6 , el modelo conjunto presenta la unión de las políticas de ambos modelos y se vuelven un único sistema.

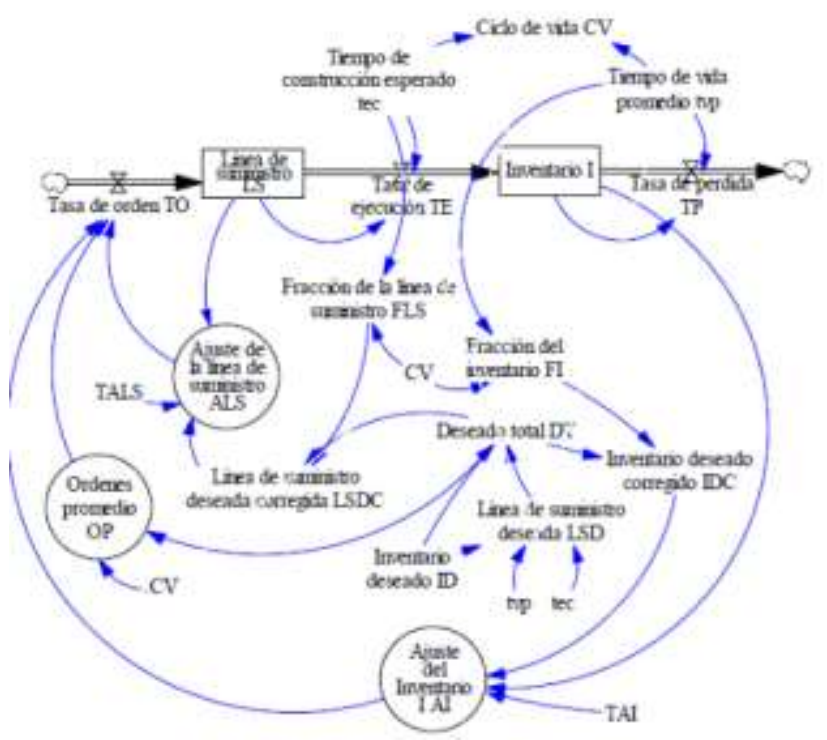

Figura 6. Modelo conjunto I+LS

A partir de los modelos de Sterman, I+LS y el modelo conjunto, los resultados permiten determinar de acuerdo al nivel de inventario deseado (ver figura 7), que el modelo I-LS, es decir, en donde se establecieron independientemente las políticas para cada variable de nivel, es aquel que logra llegar más rápido al nivel deseado del inventario en comparación a los otros dos modelos. Esto da significancia de lo importante de establecer políticas independientes para cada una de las variables, en este caso inventario y línea de suministro, ya que evidencia su comportamiento con respecto al nivel del inventario deseado.

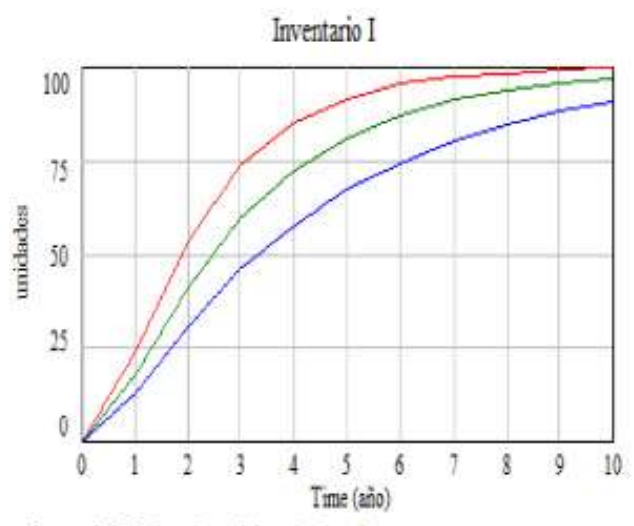

Invetiario I: Cadena de suninistro (conjunto)

Invetario I: Inventario-Lirea de sunienistro

Invertario I: Cadera de sueinistro Sternar

Figura 7. Resultados del inventario deseado. Fuente: [32]
Los modelos de inventarios, utilizando dinámica de sistemas, permiten simulaciones en el tiempo, de modo que muestren su comportamiento de acuerdo a las variables que en un determinado caso se quieran analizar. En el estudio presentado en [33], se establecen varios modelos simulados en Vensim, en donde a partir de un diagrama de Forrester, se muestra el comportamiento del inventario de acuerdo a la producción y los pedidos que se hagan, bajo ciertos factores que afectar la variable de nivel de inventario. En este caso el modelo es simulado de manera exponencial con un funcionamiento matemático, regido por una ecuación diferencial que determina el punto de equilibrio del inventario. Este modelo exponencial, como se muestra en la figura 8 , es una representación básica del funcionamiento del inventario, quien está determinado por una tasa de producción como variable de flujo de entrada y los pedidos como una variable de flujo de salida.

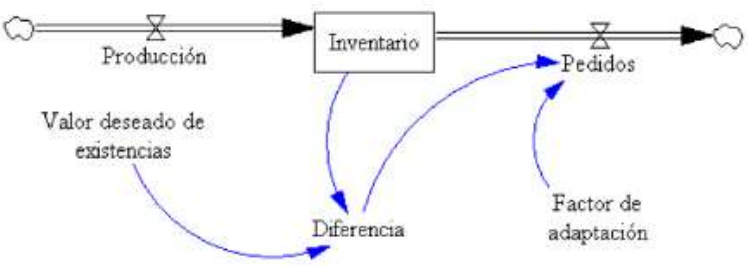

Figura 8. Diagrama de Forrester del modelo de inventario exponencial. Fuente: [33]

Este modelo, permite establecer el valor del inventario haciendo una diferencia entre la cantidad que se tiene y aquellas unidades que salen por medio de los pedidos, de manera que sea posible determinar la cantidad deseada de existencias. Esto indica que la diferencia entre el inventario y los pedidos debe estar en un valor cercano al deseado. Con este modelo simple se pretendía entender el funcionamiento de la dinámica de sistemas en la gestión de inventarios. La gestión dinámica de existencias, también permite incorporar variables con las cuales es posible determinar los comportamientos a lo largo del tiempo de parámetros que influyen en la gestión de inventarios. En este mismo estudio, el modelo presentado en la figura 9, muestra la gestión de existencias, aplicado específicamente a productos como cajas de aceite. Este modelo sigue las siguientes ecuaciones, con las cuales el modelo es dinámico y permite expresar su comportamiento y cambios en el tiempo:

$$
\text { Cajas de Aceite }(t)=\int(\text { Producción }- \text { Clientes }) d t
$$

La ecuación 3, presenta el cambio de la variable de nivel con respecto al tiempo, definida por la variable de flujo de entrada menos la variable de flujo de salida. Producción está determinada por el ajuste del inventario y la demanda de los productos y los clientes es equivalente a la solicitud con que se venda el producto. 


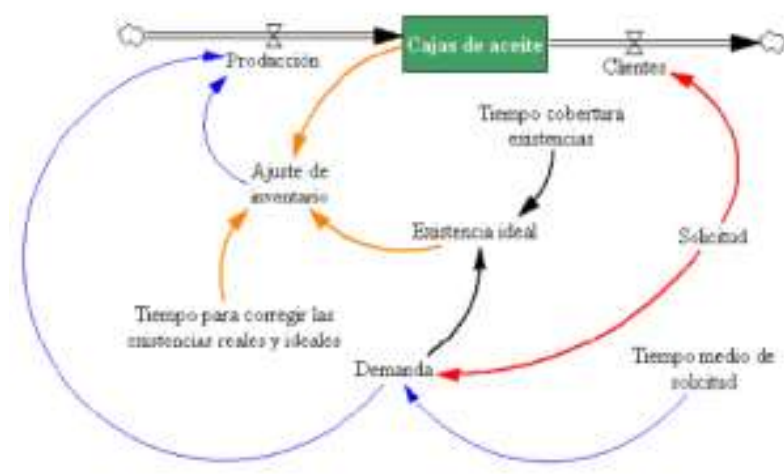

Figura 9. Modelo de gestión de existencias. Fuente: [33]

Los resultados esperados para este modelo, buscan presentar datos del comportamiento de las existencias del almacén a lo largo de un año. Esto permite determinar las cantidades necesarias en los tiempos establecidos de acuerdo a las políticas de funcionamiento del inventario. En este caso en particular, el modelo permitió conocer la cantidad de productos entre los cuales es posible atender las necesidades de los clientes sin comprometer un aumento en los costos. Es evidente que la dinámica de sistemas permite conocer de manera simulada, de acuerdo a las condiciones del modelo, los comportamientos que presenta el inventario. Es importante mencionar que el modelo es sencillo y se hace necesario considerar otras variables de tal modo que la situación se acerque mucho más a la realidad y por tal motivo su aplicación y resultados estén cercanos a los resultados esperados. En el modelo, es necesario hacer ajustes en cuanto a los tiempos de retraso que se presentan en la producción y de igual forma es importante reconocer el pronóstico en la gestión de inventario.

La toma de decisiones en el inventario, se convierten en una forma de relacionar y conectar procesos y ejecuciones entre sí, de modo que se pueda ver como hay afectaciones de los procesos entre sí. De este modo es posible mantener los costos de manejo de inventario equilibrados y por tanto la cadena de abastecimiento no se vea afectada. De acuerdo con esto, en el estudio presentado en [34], se muestra un modelo basado en "EI juego de la cerveza" creado por profesores del MIT, utilizando ecuaciones tomadas de un artículo que presenta el diseño del comportamiento del inventario de acuerdo a las decisiones tomadas y así realizar un análisis de cada uno de los modelos. Para cada una de las variaciones del modelo general se tiene una ecuación específica con la cual es posible analizar el comportamiento. Para el desarrollo del artículo, se presentan dos tipos de patrones de demanda: Step Up y Step Up Down, con tiempos de retrasos discretos y exponenciales. Con esto se busca analizar varios modelos, con los cuales sea posible determinar el comportamiento del inventario, a partir de un modelo general, como el que se observa en la figura 10 , adaptado de iThink a Vensim para una mayor comprensión. A continuación, se muestran las ecuaciones generales del modelo:

$$
I(t)=\int \operatorname{Inventario}(t . d t)+(\text { Recepción }- \text { Entrega }) d t
$$

Donde

$$
\begin{gathered}
\text { Inventario }=\text { coeficiente de inventario X Entrada } \\
\text { Recepción }=\frac{\text { Linea de suministro }}{\text { tiempo de retardo }} \\
\text { Entrega }=\text { Demanda }
\end{gathered}
$$

A partir de las ecuaciones 4, 5, 6 y 7 se empiezan a hacer modificaciones al patrón de entrada de acuerdo a cada modelo, teniendo en cuenta las combinaciones mencionadas anteriormente con relación al patrón de demanda y los tiempos de retraso. 


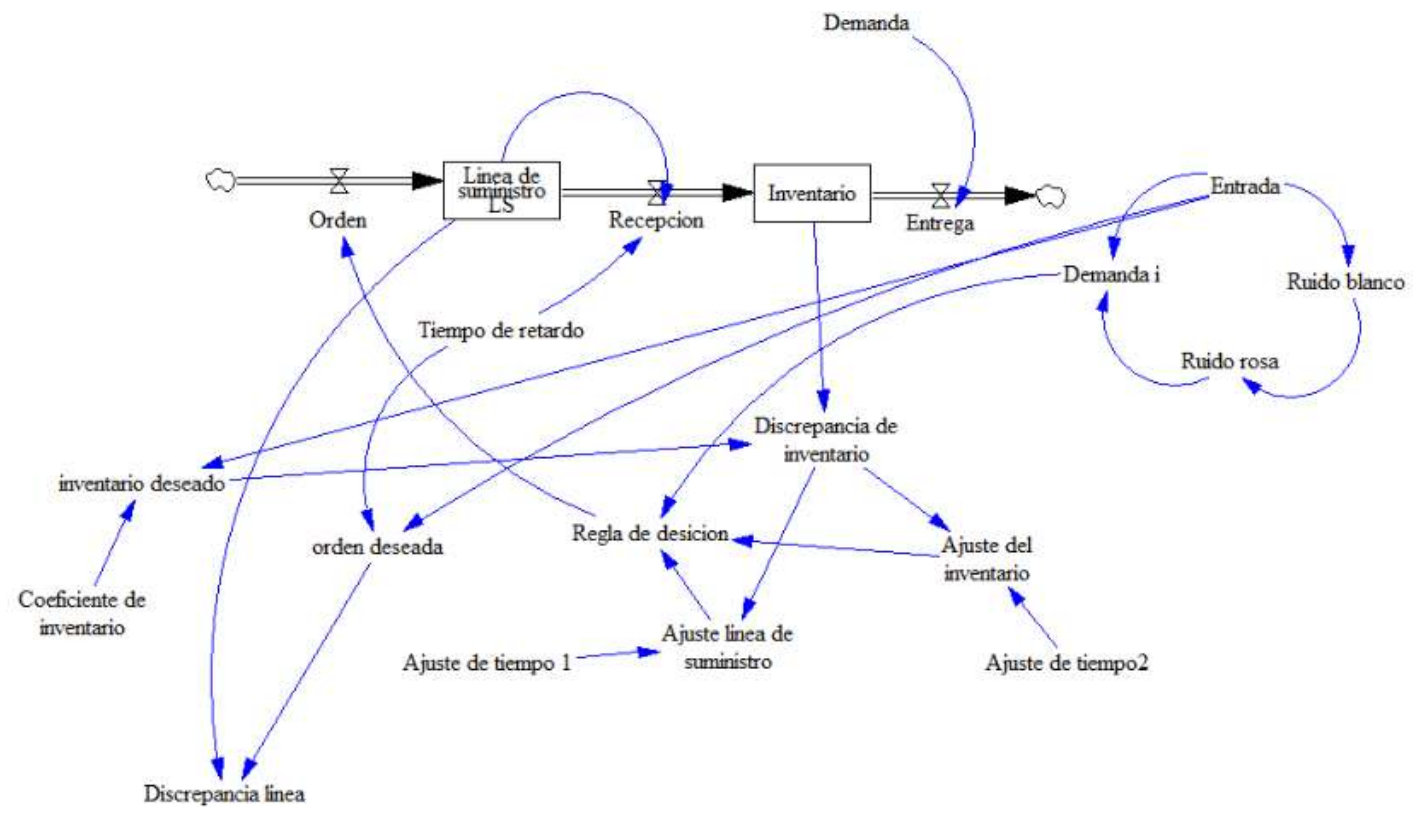

Figura 10. Modelo de inventarios adaptado de iThink a Vensim. Fuente: [34]

Los resultados obtenidos a partir de este modelo, tienen en cuenta varios factores en relación a los patrones de demanda, es decir, cómo afectan al inventario los patrones Step Up o Step Up Down. De igual forma es posible determinar el nivel del inventario, si es oscilatorio o se mantiene constante, lo que da significancia de los límites del nivel del inventario. A partir de esto es posible concluir que los patrones de demanda tienen impactos significativos en los resultados, además de esto se recuerda la necesidad de incluir los retrasos. Este tipo de modelos computacionales demuestran la viabilidad de manejar las órdenes y cumplir satisfactoriamente con los pedidos de los clientes sin caer en pedidos pendientes y de esta manera se logra que los resultados sean más eficientes y sin mayores contratiempos.

El manejo de inventarios, desde un punto de vista académico y de aprendizaje, debe ser entendido desde una perspectiva muy amplia, en donde sea posible desarrollar análisis sobre el comportamiento del inventario, de acuerdo a las decisiones que se tomen. Esto lleva a comprender que el manejo del inventario es una tarea compleja y por tanto se hace necesario establecer mejores políticas y metodologías en donde se pueda propiciar mejores soluciones a las empresas. Con relación a esto, la dinámica de sistemas se presenta como una metodología con la cual es posible analizar y aprender sobre la gestión de inventario como se presenta en [35]. En el desarrollo de este estudio, se conoce por medio de un juego, la enseñanza de la administración de inventarios con demanda estocástica e independiente, de modo que sea posible conocer la dinámica del modelo de acuerdo a la cantidad económica de pedido (EOQ). El desarrollo de este modelo se presentó en iThink, software especializado en la creación de diagramas causales, en donde es posible representar las relaciones entre varias variables, y así lograr conocer el comportamiento a través del tiempo de dichas variables. Con esto se busca que en cursos logísticos y de producción se presente la información teórica sobre inventarios, y a partir de casos reales se puedan simular modelos y tomar decisiones. Es importante mencionar que en el desarrollo de estas actividades se tienen en cuenta los fundamentos de la dinámica de sistemas para poder desarrollar cada uno de los modelos, aun así, al ser un proyecto académico, se omiten variables importantes que deben ser tenidas en cuenta.

Dentro de este estudio, se menciona la importancia de la dinámica de sistemas como herramienta base que posibilita mejorar las condiciones sobre el comportamiento del inventario, logrando determinar que cuando las empresas utilizan este tipo de metodologías, es posible tomar decisiones de acuerdo a las variables que se desee analizar. De acuerdo con esto, es posible determinar una gestión de stocks, y la realimentación de las variables según sea el caso.

En la gestión de inventarios, se explica el funcionamiento del modelo de cantidad económica de pedido, con el cual es posible determinar la demanda de un producto, siempre y cuando sea constante, uniforme y conocida [36][37]. A partir de este modelo se puede establecer una política de inventario, como la que se muestra en el trabajo desarrollado en [38]. En éste se busca analizar el comportamiento de las existencias de acuerdo a una variable de entrada, compras; y una variable de salida, ventas. Este modelo es simulado en Vensim, donde es posible relacionar las variables de flujo teniendo en cuenta el stock de seguridad. Este modelo sencillo evidentemente presenta dificultades cuando se intenta determinar el comportamiento de compras y ventas. De acuerdo a esto es necesario, que, para el stock de seguridad al ser invariable, se tenga que dar un valor inicial para el resto de la simulación. 
Para dar solución a los problemas del modelo inicial, se propone el modelo presentado en la figura 11. En este diagrama se observa cómo ya no solo son las variables de salida y entrada, sino que también entran a alimentar al modelo variables auxiliares como los días hábiles, el número de pedidos y la cantidad económica de pedido representada como $Q$ óptimo. Es importante mencionar que los datos de funcionamiento del modelo, son extraídos de un problema típico planteado en [39], con la finalidad de dar funcionamiento al modelo y así poder determinar una análisis y conclusiones con relación al modelo.

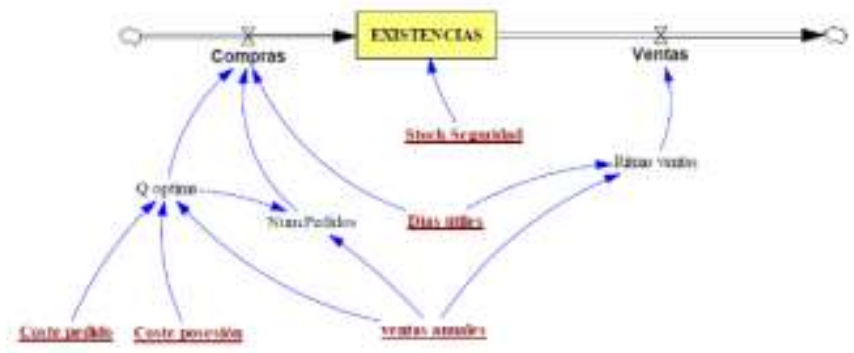

Figura 11. Diagrama de Forrester de modelo de gestión de existencias. Fuente: [38]

De acuerdo a los resultados presentados en el desarrollo del proyecto, y los datos introducidos a las variables auxiliares del modelo, es posible mencionar que se hace necesario incluir los costos de almacenamiento y de pedido, pues estos también afectan el control de las existencias. Es necesario que, dentro del modelo, y del manejo de la herramienta se incluyan funciones de desfase y aleatoriedad tales como DELAY y RANDOM, donde se pueda dar cumplimiento y simular que la demanda sea constante y que pueden existir retrasos en los plazos de entrega. Esto lleva a que el modelo sea mucho más ajustado a la realidad y pueda tener mejores resultados.

Dentro de los factores que se han mencionado anteriormente en la gestión de inventarios, es necesario hacer énfasis en los pronósticos. Estos ayudan a que el nivel del inventario se mantenga y se puedan minimizar los costos del mismo. Los pronósticos están determinados por la demanda, cuando estos son mayores que la demanda no hay ninguna preocupación, puesto que es posible satisfacer al cliente sin ningún problema. De acuerdo al comportamiento de la demanda, es recomendado tener un modelo de pronóstico, sobre todo cuando se pueda presentar un crecimiento de la demanda y esto a su vez pueda llegar a aumentar las órdenes, los costos fijos y costos de producción y compra [40].

La dinámica de sistemas, también permite incluir este tipo de variables en los modelos de inventarios, ya que los pronósticos tienen un alto impacto en los resultados económicos de las empresas y ayudan a prever la demanda de una manera realista [41]. Es muy común que en las organizaciones se presenten problemas de excesos y faltantes. Esto lleva a que los pronósticos estén involucrados dentro de este problema. Es por esto que en el trabajo desarrollado en [42], se realiza un estudio entre los excesos y faltantes con relación al inventario, teniendo en cuenta la demanda real y la demanda pronosticada, con la finalidad de comparar y analizar la funcionalidad de los pronósticos dentro de la gestión de inventarios. Dentro de éste documento se evalúan varios modelos de pronósticos como regresión lineal simple, suavización exponencial simple y doble, teniendo en cuenta el modelo presentado en la figura 12, en donde es posible observar los bucles de realimentación del sistema y la relación entre cada una de las variables.

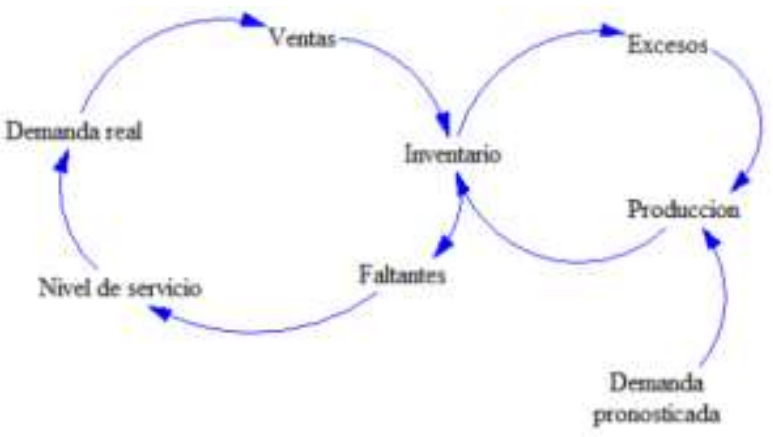

Figura 12. Diagrama causal para el modelo presentado en [42]

Particularmente, el estudio se centra en los pronósticos de la demanda, los cuales van a afectar directamente a la producción y relacionado a esto se producirán cambios en el inventario generando así excesos o faltantes. Con esto es posible concluir la importancia de los pronósticos en los modelos de inventarios con dinámica de sistemas. Además de esto se hace necesario incluir varios criterios como los costos de mantener y de pedir, al igual que los costos por faltantes y excesos los cuales no tienen siempre el mismo valor.

Con la suavización exponencial doble, se logra que con este sistema de pronóstico, la demanda pronosticada con respecto a la demanda real sea muy cercanas y permita tener mayores variaciones con relación a los datos. Esto da significancia de que se puede tener un control sobre los excesos y faltantes de modo que no se vea afectado el nivel de servicio.

En el desarrollo del documento, como se ha venido mencionando, es posible resaltar la dinámica de sistemas en el estudio y análisis del comportamiento de los inventarios, ya que gracias a esta metodología es posible identificar y relacionar varios elementos con los cuales es posible construir un modelo robusto que presente resultados cercanos a la realidad, y con éste sea posible mejorar las políticas y condiciones en el proceso de gestión de inventarios. Para desarrollar un modelo, es necesario conocer la relación entre las variables. En el modelo presentado en [43], hay una relación de causalidad entre variables que afectan el inventario por medio de una hipótesis dinámica en el control de inventarios. Dentro de estas variables es 
posible encontrar: ventas, producción, inventario, como variables de nivel y costos, materia prima, utilidades, entre otras, actuando como variables de flujo y auxiliares.

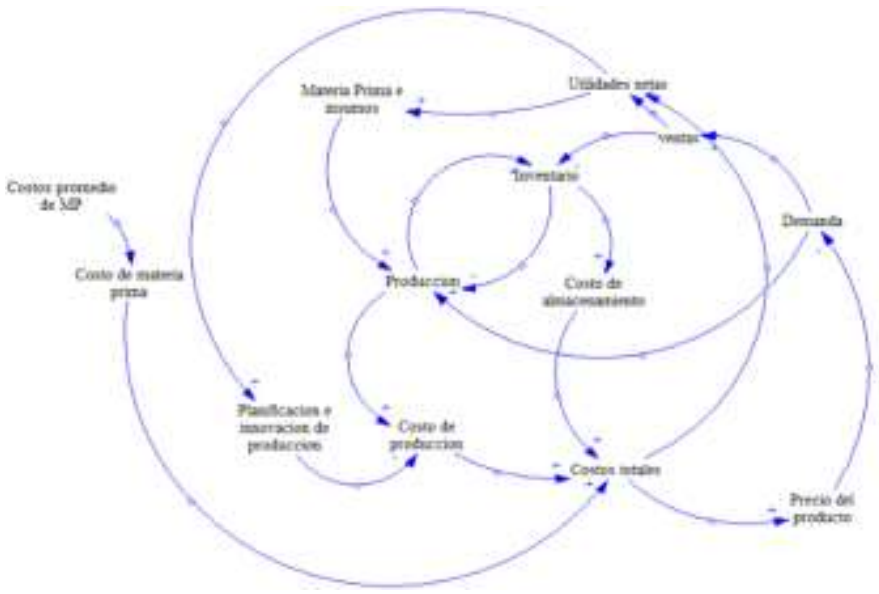

Figura 13. Diagrama causal de un sistema de inventario. Fuente: adaptado de [43]

Como se observa en la figura 13 , es posible relacionar variables que afectan el inventario de manera directa 0 indirecta. A partir de este diagrama se pueden establecer hipótesis dinámicas como las que se mencionan en el trabajo en donde la producción se ve afectada por el nivel de inventario y por materia prima, dentro de las cuales se incluyen disponibilidad, costos, compras, entre otros.

Los costos totales están formados por los costos de producción, materia prima y almacenamiento y esto lleva a que los costos totales impacten en las utilidades netas y en el precio del producto, que este último a su vez afectará la demanda de los productos y las ventas. Con el diagrama causal es posible identificar los bucles de realimentación del sistema, con el cual se establecen relaciones entre las variables y a partir de esto analizar el comportamiento de cada una de estas.

Como se mencionaba anteriormente, las variables de nivel del modelo son el inventario, las ventas $y$ producción. Los resultados obtenidos en el modelo, afirman la potencialidad de la dinámica de sistemas en la gestión de inventarios como ayuda metodológica en la formulación y el análisis de control y manejo de los inventarios.

De manera más específica, con relación al modelo presentado, es posible mencionar que cuando el inventario supera la estabilidad promedio, inmediatamente el precio va disminuir considerablemente. Asociado al precio, cuando éste cambia, el nivel de ventas también se verá afectado en el tiempo. Además, cuando los costos de materia prima se incrementan, estos van a afectar directamente en la producción y las ventas, y por tanto al inventario.

En general, cada uno de los modelos presentados, utilizan la dinámica de sistemas para analizar el comportamiento del inventario. Sin embargo, cada uno de ellos plantea formas diferentes de relación entre las variables, lo cual se justifica desde el caso de estudio que se pretende analizar. Aun así, es necesario que en muchos de los modelos se incluyan los pronósticos, ya que estos impactan positivamente en el control y los costos del inventario.

Es importante que las empresas entiendan la necesidad de analizar los inventarios y la manera de controlarlos para mejorar las políticas internas y así crear ventajas competitivas y prestar servicios de mayor calidad. Con herramientas como la DS, las empresas pueden validar sus políticas, evaluar el comportamiento de las variables que se están incluyendo en el inventario, pero también es posible observar como es el impacto en el resto de las actividades de producción de la cadena de suministro.

\section{Conclusiones}

El proceso de gestión de inventarios requiere de un modelo que adquiera la facilidad atender las necesidades de los cliente, teniendo en cuenta factores y variables. Estos factores pueden relacionarse con la producción, demanda, ventas, pérdida de productos, tiempos de espera, unidades rechazadas, nivel de servicio, entre otras más. También es posible que el modelo tenga en cuenta los pronósticos de la demanda en conjunto con las demás variables mencionadas. La formulación del modelo matemático o de ecuaciones que soporta el modelo dinámico, debe ser un proceso basado en el comportamiento del inventario teniendo presente los conceptos teóricos más importantes relacionados con el manejo y control del inventario.

La dinámica de sistemas como herramienta para el análisis de gestión de inventarios, permite presentar buenos resultados siempre y cuando los modelos se establezcan de la manera más adecuada, relacionando las variables y observando su comportamiento en el tiempo, por medio de realimentaciones que llevan a estudiar un determinado problema. Se resalta la importancia de los bucles de realimentación de los sistemas, pues esto garantiza que el sistema tenga flujo y sea dinámico.

Con relación a los modelos presentados, se concluye la importancia de establecer políticas independientes para las variables de nivel, ya que con esto es posible que el comportamiento del inventario con respecto al nivel deseado, tenga mejores resultados que haciendo una evaluación de políticas conjunta. Asociado a esto, también es necesario que los modelos sean mucho más robustos en donde se incluyan más variables, ya que la dinámica de sistemas lo permite desde sus fundamentos teóricos. Esto lleva a que los modelos puedan crecer mucho más y los resultados sean mucho más cercanos a la realidad, ya que cuando los modelos son muy sencillos los resultados esperados pueden tener muchas incoherencias. Para esto es necesario incluir tiempos de retrasos y espera, ya que estos afectan directamente a la productividad y de manera general, a todos los procesos de la empresa. 
Los patrones de demanda, pueden cambiar y afectar directamente al inventario, es por ello que se hace necesario mantener un nivel de inventario constante y no sobrepasar los límites, puesto que se incurriría en otros costos. Además de esto es necesario recurrir a modelos computacionales en donde se garantice el cumplimiento satisfactorio de las órdenes y pedidos de tal modo que el nivel de satisfacción del cliente se mantenga y se logren mejores resultados sin contratiempos.

Con este tipo de herramientas se posibilita el mejoramiento de las condiciones en el manejo y control del inventario, haciendo posible determinar una gestión de stocks y una realimentación entre las variables. Esto lleva a que metodológicamente sirva como herramienta de enseñanza en asignaturas donde se relacionen conceptos de inventario y sea posible tomar decisiones frente a los comportamientos de las variables que impactan en el inventario. Dentro de estas variables es importante analizar los costos totales y la manera en que estos impactan en la producción y ventas. Los costos de almacenamiento y pedido, de acuerdo con varios de los modelos analizados, es necesario incluirlos dentro de los modelos, ya que estos afectan directamente el control de existencias.

Con relación al manejo de las herramientas como iThink y Vensim, es necesario incluir funciones como DELAY y RANDOM, con la finalidad de incluir los retrasos en los plazos de entrega, y de este modo tener un modelo mucho más robusto y cercano a la realidad.

Los pronósticos afectan directamente la producción en una empresa, y estos a su vez cambios en la demanda y por tanto impactan en el control del inventario. Por esto, hay que incluir los pronósticos dentro de los modelos, y así tener un control en los excesos y faltantes que en la mayoría de casos es generado por los pronósticos.

Cada uno de los modelos presentados y analizados, tienen aspectos en común. Esto se justifica desde la teoría que rige el control y gestión del inventario. Es muy claro que cada uno de los modelos estudiados en este documento tiene algunas particularidades que los diferencian de los demás. En cada uno de ellos se evidencia el comportamiento e influencia de las variables en el inventario o existencia. Los costos del manejo de los inventarios están intrínsecos en el funcionamiento de cada uno de los modelos, pero, aun así, no se evidencian aspectos importantes relacionados con la minimización de los costos de modo que se pueda contribuir con la cadena de suministro.

\section{Trabajos futuros}

Proponer un modelo, que incluyan estrategias de mejoramiento de los inventarios, utilizando la dinámica de sistemas como metodología de desarrollo, la cual permita representar un modelo con relaciones de causalidad entre las variables, teniendo en cuenta la importancia de incluir los pronósticos en el modelo, además de los costos de almacenamiento y pedido, y finalmente los retrasos que se presentan en las entregas, entre otras más variables de investigación que a futuro deben incluirse a fin de mejorar el proceso de gestión y manejo del inventario.

\section{Referencias}

[1] Donald J. Bowersox, David J. Closs, and M. Bixby Cooper, Administracion y logistica en la cadena de suministros. 2007.

[2] S. lopez Ricardo, "Cadena De Suminstro," Universidad Autonoma de Nuevo Leon., 2001.

[3] J. L. Pulido C, "Gestión de la cadena de suministro. El último secreto," p. 23, 2014.

[4] R. H. Ballou, Logistica: Administración de la cadena de suministro, vol. 1. 2008.

[5] El Empresarismo, "Mala gestión de inventarios causas quiebre de las pymes | EL EMPRESARIO." [Online]. Available: http://elempresario.mx/guia-util/mala-gestioninventarios-causas-quiebre-pymes. [Accessed: 01-Sep2017].

[6] Yaditza del sol Gonzalez, "De inventarios y cadenas de suministros..., para no perder la logística > Economía con tinta , Granma - Órgano oficial del PCC." [Online]. Available: http://www.granma.cu/economia-con-tinta/2017-08-24/deinventarios-y-cadenas-de-suministros-para-no-perder-lalogistica-24-08-2017-23-08-01. [Accessed: 01-Sep-2017].

[7] M. Duque, J. Osorio, and D. Agudelo, "Los inventarios en las empresas manufactureras, su tratamiento y su valoración. Una mirada desde la contabilidad de costos.," Contaduria Univ. Antioquia, vol. 56, pp. 61-79, 2010.

[8] "Inventarios de empresas en EU logran en junio su aumento más grande | Televisa News." [Online]. Available: http://noticieros.televisa.com/ultimas-noticias/eeuu/2017-0815/inventarios-empresas-eu-logran-junio-su-aumento-masgrande/. [Accessed: 01-Sep-2017].

[9] L. C. Global, “- Estructura de Contenidos -," pp. 1-16.

[10] "Gacela MisiónPyme, el premio para los emprendedores | Emprendimiento | Negocios | Portafolio." [Online]. Available: http://www.portafolio.co/negocios/emprendimiento/gacelamisionpyme-el-premio-para-los-emprendedores-509241. [Accessed: 01-Sep-2017].

[11] "Proteger la caja y bajar inventarios, la meta de Fabricato al cerrar su planta por 15 días." [Online]. Available: http://www.dinero.com/empresas/articulo/fabricato-razonespor-las-que-suspende-su-operacion-15-dias/248587. [Accessed: 01-Sep-2017].

[12] J. Aracil, Dinámica de Sistemas, Edison. Madrid.

[13] J. D. Sterman, Systems Thinking and Modeling for a Complex World, vol. 6, no. 1. 2000.

[14] G. Arbeláez Ordóñez, P. Vargas, O. Herminda, R. Muñoz-, G. Arbeláez, and C. Ordóñez, "Integral management from a strategic planning model perspective," vol. 9, no. 2012, p. 28, 2012.

[15] P. P. M. COALLA, UF0476 - Gestión de inventarios: Ediciones Paraninfo, S.A, 2017.

[16] J. A. Zapata Cortes, Fundamentos de la gestión de inventarios. 2014.

[17] J. Heizer and B. Render, Dirección de la Producción y de Operaciones. 2015.

[18] J. W. Forrester, "Diseñando el futuro," pp. 1-11, 1998.

[19] J. P. Aljure León, "Pensamiento sistémico: la clave para la creación de futuros realmente deseados," Elegir, vol. 9, pp. 8-16, 2007. 
[20] B. Angerhofer and M. Angelides, "System dynamics modelling in supply chain management: research review," 2000 Winter Simul. Conf. J., pp. 342-351, 2000.

[21] K. Ogata, Dinámica de Sistemas. 1987.

[22] J. Aracil and F. Gordillo, "Dinamica de Sistemas.pdf." 1997.

[23] J. A. Sera Uran, Conrado Augusto; Arango Serna, Martin Dario; Gomez Lizarazo, Dinamica de sistemas para la evaluacion de corredores logisticos. Medellin, 2016.

[24] "De diagramas causales a diagramas de flujos y niveles Flujos y niveles Diagrama causal de la venta de esposas en Haryana."

[25] S. Jaén, "Ayudas para la elaboraci ' on de diagramas causales," pp. 1-9.

[26] R. M. Chavez Guillén, "Dinamica de sistemas," Diagramas causales, pp. 1-49, 2010.

[27] AECA Asociación Española de Contabilidad y Administración de Empresas, Aplicación de la Contabilidad de Gestión a la Cadena de Suministros. 2007.

[28] FIAEP, "Control y Manejo de Inventario y Almacén," Fund. Iberoam. Altos Estud. Prof. FIAEP, vol. 1, pp. 1-59, 2014.

[29] D. E. Hidalgo and A. O. Angeles, "Control y evaluación de inventarios en tiendas de autoservicio," Universidad Autonoma del Estado de Hidalgo., 2007.

[30] J. W. Forrester, Urban Dinamics, Pegasus co. 1999.

[31] B. Múgica and B. Gonzáles, "La Dinámica de Sistemas como Metodología para la elaboración de Modelos de Simulación," p. $41,1998$.

[32] F. G. Avellaneda, F. A. V. Rozo, and M. J. C. Ayala, "Sobre La Dinámica de un Sistema de Gestión de Inventarios," $9^{\circ}$ Encuentro Colomb. Dinámica Sist., 2011.

[33] M. Xu, "Modelos de gestión de existencias de una empresa a través de dinamica de sistemas.," pp. 1-82, 2014.
R. . Ramirez. A, Fernandez.J, "Analisis de la toma de decisiones en el manejo de inventarios utilizando dinámica de sistemas.," X Congrso Latinoam. Din. Sist., pp. 1-49, 2012.

[35] G. C. González, U. Eafit, J. C. Urrego, U. Eafit, and S. R. Echeverri, "Juesgo empresarial para el aprendizaje de inventarios.," pp. 1-10, 2009.

[36] R. Montenegro Lopez, "Diseño e implementacion de un sistema de inventarios, aplicando simulacion montecarlo, en una empresa de servicios petroleros," 2008.

[37] E. C. Rodríguez, "Modelo de inventarios para control económico de pedidos en empresa comercializadora de alimentos," Rev. Ing. Univ. Medellín, vol. 14, no. 27, pp. 163178, 2015.

[38] X. Canaleta, "Estudio desde el punto de vista de teoria de sistemas del modelo de Wilson para la gestion de inventarios."

[39] P. Llorente, Casani. F A, Economía y organización de empresas. 1997.

[40] R. Alberto, S. Andr, and J. Jos, "Application of Forecast Models in Products of Massive Consumption," Biotecnol. en el Sect. Agropecu. y Agroindustriall, vol. 10, no. 2, pp. 117125, 2012.

[41] J. A. Arango Marin, J. A. Giraldo Garcia, and O. D. Castrillón Gómez, "Gestión de compras e inventarios a partir de pronósticos Holt-Winters y diferenciación de nivel de servicio por clasificación ABC Inventory and buy management from Holt-Winters forecasting and service level discrimination by ABC classification," Sci. Tech. Año XVIII, vol. 18, no. 4, pp. 743-747, 2013.

[42] C. A. Pretel, O. Galvis, L. Rendon, and J. Osorio, "Dinámica de sistemas para la selección de un sistema de pronóstico con base en el impacto de excesos y faltantes," Sist. Telemàtica, vol. 11, no. 24, pp. 55-71, 2013.

[43] F. L. Martínez and J. G. V. Oquendo, "A System Dinamics Model For Inventory Management," Rev. Soluciones Postgrado, vol. 6, no. 11, pp. 121-135, 2014. 